\title{
Reproducibility of twitch mouth pressure, sniff nasal inspiratory pressure, and maximal inspiratory pressure
}

\author{
J.O. Maillard*, L. Burdet*, G. van Melle**, J.W. Fitting*
}

Reproducibility of twitch mouth pressure, sniff nasal inspiratory pressure, and maximal inspiratory pressure. J.O. Maillard, L. Burdet, G. van Melle, J.W. Fitting. OERS Journals Ltd 1998.

ABSTRACT: Twitch mouth pressure $(P$ mo,tw $)$ during magnetic phrenic nerve stimulation and sniff nasal inspiratory pressure (SNIP) were recently proposed as alternative noninvasive methods for assessing inspiratory muscle strength. This study aimed to compare their reproducibility with maximal inspiratory pressure (MIP) in normal subjects.

Ten healthy subjects were studied at functional residual capacity in semirecumbent position. Cervical magnetic phrenic nerve stimulation was performed during gentle expiration against an occlusion incorporating a small leak. Constancy of stimulation was controlled by recording diaphragmatic electromyogram. Within and betweensession reproducibility of pressure were studied for $P$ mo,tw, SNIP, and MIP. The subjects were studied during a session of 10 manoeuvres repeated after 1 day and 1 month.

The mean values were $16 \mathrm{cmH}_{2} \mathrm{O}$ for $P$ mo,tw, $118 \mathrm{cmH}_{2} \mathrm{O}$ for $\mathrm{SNIP}$, and $115 \mathrm{cmH}_{2} \mathrm{O}$ for MIP. For the three tests, the within subject variation was small in relation to between-subject variation, with the intraclass correlation coefficient ranging 0.790.90 for $P$ mo,tw, 0.85-0.92 for SNIP, and 0.88-0.92 for MIP. At 1 day interval, the coefficient of repeatability ( $2 \mathrm{sD}$ of differences) was $3.6 \mathrm{cmH}_{2} \mathrm{O}$ for $P_{\mathrm{mo}, t w}, 32 \mathrm{cmH} O \mathrm{O}$ for SNIP and $28 \mathrm{cmH}_{2} \mathrm{O}$ for MIP. At 1 month interval, the coefficient of repeatability was $5.8 \mathrm{cmH}_{2} \mathrm{O}$ for $P \mathrm{mo}_{\text {,tw }}, 23 \mathrm{cmH}_{2} \mathrm{O}$ for SNIP and $21 \mathrm{cmH}_{2} \mathrm{O}$ for MIP.

We conclude that the within session reproducibility of the new tests twitch mouth pressure and sniff nasal inspiratory pressure is sufficient to be clinically useful. For sniff nasal inspiratory pressure, the between session reproducibility established after 1 day was maintained after 1 month. For twitch mouth pressure, the between session reproducibility declined slightly after 1 month. These characteristics should be considered when using these methods to follow an individual patient over time. Eur Respir J 1998; 11: 901-905.
*Division de Pneumologie, Centre Hospitalier Universitaire Vaudois and **Institut Universitaire de Médecine Sociale et Préventive, Lausanne, Switzerland.

Correspondence: J.W. Fitting

Division de Pneumologie

CHUV

CH-1011 Lausanne

Switzerland

Fax: 41213141384

Keywords: Diaphragm phrenic nerve stimulation respiratory muscles respiratory pressures

Received: June 171997

Accepted after revision January 161998
The measurement of mouth pressure during a maximal inspiratory effort against a quasi occlusion (maximal inspiratory pressure (MIP)) is classically established as the standard for assessment of inspiratory muscle strength [1]. However, in addition to being closely dependent on subject collaboration, this manoeuvre is demanding and unpleasant. Thus, whilst high values of MIP exclude inspiratory muscle weakness, lower values are frequently difficult to interpret, reflecting either a true muscle weakness or a lack of motivation and co-ordination [2].

A recent alternative noninvasive method consists of performing a short maximal sniff through one nostril while measuring nasal pressure through a plug occluding the contralateral nostril (sniff nasal inspiratory pressure (SNIP)) [3-5]. This natural manoeuvre is easier and less unpleasant than the static effort of MIP, but nevertheless depends on volitional muscle contraction. The need for a nonvolitional test, especially in situations where collaboration is totally lacking, has led to the development of phrenic nerve stimulation. Because it is better tolerated and easier to apply, cervical magnetic stimulation has now largely replaced electrical stimulation $[6,7]$. The measurement of twitch mouth pressure $(P$ mo,tw $)$ during magnetic stimulation was recently reported as a reliable assessment of diaphragm strength [8].

Thus, several noninvasive techniques are now available for assessing inspiratory muscle strength, based on volitional and nonvolitional manoeuvres. In order to use these new methods in clinical settings, it is necessary to characterize them in comparison with the established reference method. The aim of this study was to determine the reproducibility of SNIP and Pmo,tw during cervical magnetic stimulation and to compare them to classical MIP in a group of normal subjects.

\section{Materials and methods}

\section{Study subjects}

Ten healthy volunteers (nine males, one female) were studied. Their characteristics were (mean \pm SD): age $28 \pm 7$ 
yrs; weight $67 \pm 9 \mathrm{~kg}$; and height $1.76 \pm 0.08 \mathrm{~m}$. None of them reported rhinitis, previous nasal surgery or respiratory or neuromuscular disease. Anterior rhinoscopy was performed in each of them to exclude any major anatomical abnormality. Two subjects were familiar with the SNIP and MIP manoeuvres, the other eight were not. None of the subjects were familiar with cervical magnetic stimulation.

All subjects had a forced vital capacity (FVC) $>80 \%$ and a forced expiratory volume in one second (FEV1)/FVC ratio $>85 \%$ of predicted values. The protocol was approved by the ethics committee of our hospital and all subjects gave their informed consent.

\section{Methods}

The FVC and FEV1 were measured by a mass flow sensor (SensorMedics 6200 Autobox, Yorba Linda, CA, USA).

The phrenic nerves were bilaterally activated with cervical magnetic stimulation using a Magstim 200 with a circular $90 \mathrm{~mm}$ coil and a maximal output of 2 Tesla (Madaus Medizin-Elektronik Gundelfingen, Germany). The coil was placed on the back of the flexed neck parallel to the frontal plane of the subject with the central hole over the spinous processes. To optimize the position of the coil in each subject, several initial stimulations were perfomed at $60 \%$ of maximal output between C6 and C7, until the largest diaphragmatic electromyographic response could be elicited. Thereafter, this site was marked and all stimulations were performed at $100 \%$ of the stimulator output. The stimulations were applied near functional residual capacity (FRC) (see below) during quiet breathing, as judged from visual inspection of chest wall movements.

The electromyogram (EMG) of the diaphragm was recorded with bilateral surface skin electrodes placed over the seventh intercostal spaces on the anterior auxiliary line, with the reference electrode on the eighth ribs. Both EMG tracings were displayed on an oscilloscope with option of digital storage (HM 208; Hameg, Frankfurt, Germany). The EMG tracings were stored on screen after each stimulation and the amplitudes of the left and right diaphragmatic EMG responses were measured. The sum of amplitudes of left and right EMG responses was calculated for each stimulation.

$P$ mo,tw was measured using a mouthpiece connected to a pressure transducer (MicroSwitch 126PC; Honeywell, Freeport, IL, USA) via a polyethylene catheter (length 100 $\mathrm{cm}$, internal diameter $1 \mathrm{~mm})$. The mouthpiece could be nearly occluded, leaving a $1 \mathrm{~mm}$ leak. Twitch mouth pressure was measured during a gentle expiratory effort performed from FRC through the leak. This method was selected because, during phrenic nerve stimulation, glottis closure often prevents transmission of pressure changes from the thoracic cavity to the mouth [8-10]. Therefore, in a preliminary study, the pressure generated at the airway opening by cervical magnetic stimulation was measured using three different techniques. In three subjects, twitch oesophageal pressure $\left(P_{\text {oes,tw }}\right)$ was compared to either $P$ mo,tw with airway occlusion at FRC, $P$ mo,tw during a gentle exhalation from FRC, or twitch nasal pressure at FRC as measured for SNIP (see below). The Pmo,tw during gentle exhalation presented the best agreement with
Poes,tw in all subjects. Hamnegard et al. [8] also concluded that glottis closure can be avoided with this technique.

SNIP was measured through a plug occluding one nostril during a sniff performed through the contralateral nostril [3]. The plug was made of two waxed ear plugs (Calmor, Neuhausen am Rheinfall, Switzerland) hand-fitted around the tip of a polyethylene catheter (length 100 $\mathrm{cm}$, internal diameter $1 \mathrm{~mm}$ ) connected to the same pressure transducer. Prior to the manoeuvres, a topic vasoconstrictor (Otrivin 1\%, Zyma, Switzerland) was instilled in each nostril to avoid any mucosal congestion. The subjects were asked to perform maximal, short and sharp sniffs from FRC, with their mouth closed.

Tracings of $P$ mo,tw and SNIP were recorded on paper at a speed of $10 \mathrm{~mm} \cdot \mathrm{s}^{-1}$. The pressure transducers were calibrated before each study using a U-tube water manometer.

Maximal inspiratory pressure (MIP) was measured with a Mouth Pressure Meter (Chest Scientific Instruments Ltd, Westerham, UK). The subjects were asked to perform a maximal inspiratory effort from FRC during 2-3 s. The reported value is the maximal pressure averaged over $1 \mathrm{~s}$.

\section{Study design}

All measurements were performed with the subjects in semirecumbent position, i.e. lying supine with the chest and head elevated at $30^{\circ}$. Before any measurement, the subjects rested quietly for at least $15 \mathrm{~min}$ in this position while the recording equipment was installed. During the study, the subjects were asked to breathe quietly, to remain on the bed and to avoid any physical effort in order to prevent the twitch potentiation phenomenon $[11,12]$.

Each of the 10 subjects was studied during three sessions, the second session taking place after 1 day and the third after 1 month (1.5 months in two subjects because of acute rhinitis). The sessions always followed the same sequence in order to avoid the twitch potentiation phenomenon $[11,12]$. Firstly, $P$ mo,tw was measured during 10 stimulations performed at $30 \mathrm{~s}$ intervals. The mean of the sums of the left and right EMG responses was calculated for the 10 stimulations. Twitches were rejected when the EMG response was $>10 \%$ below the mean, and supplementary stimulations were performed until 10 EMG responses were within $10 \%$ of the mean. Secondly, SNIP was measured during 10 maximal sniffs performed at $30 \mathrm{~s}$ intervals. The trials were retained for analysis if the pressure tracing showed a regular upstroke and a sharp peak, with a time to peak of less than $400 \mathrm{~ms}$. Thirdly, MIP was measured during 10 manoeuvres performed at $30 \mathrm{~s}$ intervals.

\section{Analysis}

The median values were considered for $P$ mo,tw because it is a nonvolitional test, and maximal values were considered for SNIP and MIP because these two tests are volitional. The methods were compared by calculating the following ratios: $P$ mo,tw/SNIP, $P$ mo,tw/MIP and SNIP/MIP. The reproducibility of the three methods was assessed as follows. For each method, a two way analysis of variance (ANOVA) was performed, considering as independent variables the subjects on one way and the sessions on the other. In each session, the within session reproducibility 
of the three methods was assessed by calculating the intraclass correlation coefficient. This index is based on a one way ANOVA and expresses the ratio of between-subject variation to total variation [13]. In order to compare present data with previous studies, the within session reproducibility was also assessed by calculating the coefficient of variation. The between-occasion reproducibility of the three methods was assessed in the short term (1 day) between sessions 1 and 2 and in the long term (1 month) between sessions 2 and 3. The mean and SD of differences between sessions were calculated, and the coefficient of repeatability was computed as twice the SD. The coefficient of repeatability indicates the value below which $95 \%$ of differences are expected [14].

\section{Results}

During gentle exhalation from FRC, the subjects generated a slight positive mouth pressure. Considering all the values of the ten subjects, the mean mouth pressure produced just before the magnetic stimulus was $3.2(1.5) \mathrm{cm}$ $\mathrm{H}_{2} \mathrm{O}$.

$P$ mo,tw was consistently smaller than SNIP or MIP. In five subjects SNIP exceeded MIP, whereas in the other five subjects MIP exceeded SNIP. The mean pressures were $16.4 \mathrm{cmH}_{2} \mathrm{O}$ for $P$ mo,tw, $117.6 \mathrm{cmH}_{2} \mathrm{O}$ for SNIP and $115.1 \mathrm{cmH}_{2} \mathrm{O}$ for MIP. The mean ratio $P$ mo,tw/SNIP was 0.14 , with values ranging $0.08-0.20$. The mean ratio $P$ mo,tw/ MIP was 0.15 , with values ranging $0.09-0.23$. The mean ratio SNIP/MIP was 1.03, with values ranging 0.85-1.32 (table 1).

The interindividual variability was highly significant ( $\mathrm{p}<0.01)$ for $P$ mo,tw $(\mathrm{F}=139)$, SNIP $(\mathrm{F}=198)$, and MIP $(\mathrm{F}=242)$, each on nine degrees of freedom (df). The

Table 1. - Median values of twitch mouth pressure ( $P$ mo,tw), and maximal values of sniff nasal inspiratory pressure (SNIP) and of maximal inspiratory pressure (MIP)

\begin{tabular}{lcrrrrr}
\hline $\begin{array}{l}\text { Subject } \\
\text { No. }\end{array}$ & $\begin{array}{c}P \text { mo,tw } \\
\mathrm{cmH}_{2} \mathrm{O}\end{array}$ & $\begin{array}{c}\text { SNIP } \\
\mathrm{cmH}_{2} \mathrm{O}\end{array}$ & $\begin{array}{c}\text { MIP } \\
\mathrm{cmH}_{2} \mathrm{O}\end{array}$ & $\begin{array}{c}P \text { mo,tw/ } \\
\text { SNIP }\end{array}$ & $\begin{array}{c}P \text { mo,tw/ } \\
\text { MIP }\end{array}$ & $\begin{array}{c}\text { SNIP/ } \\
\text { MIP }\end{array}$ \\
\hline 1 & 13.8 & 142.7 & 123.3 & 0.10 & 0.11 & 1.16 \\
2 & 10.5 & 82.3 & 95.0 & 0.13 & 0.11 & 0.87 \\
3 & 12.5 & 117.0 & 138.3 & 0.11 & 0.09 & 0.85 \\
4 & 15.7 & 115.3 & 117.7 & 0.14 & 0.13 & 0.98 \\
5 & 18.1 & 96.0 & 111.7 & 0.19 & 0.16 & 0.86 \\
6 & 24.3 & 128.7 & 104.7 & 0.19 & 0.23 & 1.23 \\
7 & 10.0 & 118.0 & 109.0 & 0.08 & 0.09 & 1.08 \\
8 & 19.2 & 128.0 & 97.0 & 0.15 & 0.20 & 1.32 \\
9 & 22.0 & 109.0 & 101.3 & 0.20 & 0.22 & 1.08 \\
10 & 17.8 & 138.7 & 153.3 & 0.13 & 0.12 & 0.90 \\
Mean & 16.4 & 117.6 & 115.1 & 0.14 & 0.15 & 1.03 \\
SD & 4.8 & 18.5 & 18.7 & 0.04 & 0.05 & 0.17 \\
\hline
\end{tabular}

Table 2. - Intraclass correlation coefficients of twitch mouth pressure $\left(P_{\text {mo,tw }}\right)$, sniff nasal inspiratory pressure (SNIP), and maximal inspiratory pressure (MIP)

\begin{tabular}{lccc}
\hline $\begin{array}{l}\text { Session } \\
\text { No. }\end{array}$ & Pmo,tw & SNIP & MIP \\
\hline 1 & 0.90 & 0.92 & 0.88 \\
2 & 0.79 & 0.89 & 0.92 \\
3 & 0.81 & 0.85 & 0.91 \\
\hline
\end{tabular}

The intraclass correlation coefficient expresses the ratio of between subject variation to total variation. intersession variability was lower but was also significant $(\mathrm{p}<0.01)$ for $P$ mo,tw $(\mathrm{F}=11)$, SNIP $(\mathrm{F}=31)$ and MIP $(\mathrm{F}=6)$, each on two df. There was a significant interaction $(\mathrm{p}<0.01)$ between the two independent variables, subjects and sessions, for Pmo,tw $(\mathrm{F}=10)$, SNIP $(\mathrm{F}=27)$ and MIP $(\mathrm{F}=23)$, each on $18 \mathrm{df}$.

Table 3. - Between-session coefficient of repeatability of twitch mouth pressure $\left(P_{\mathrm{mo}, \mathrm{tw}}\right)$, sniff nasal inspiratory pressure (SNIP) and maximal inspiratory pressure (MIP)

\begin{tabular}{lccc}
\hline $\begin{array}{l}\text { Time } \\
\text { interval }\end{array}$ & $\begin{array}{c}P \text { mo,tw } \\
\left(\mathrm{cmH}_{2} \mathrm{O}\right)\end{array}$ & $\begin{array}{c}\text { SNIP } \\
\left(\mathrm{cmH}_{2} \mathrm{O}\right)\end{array}$ & $\begin{array}{c}\mathrm{MIP} \\
\left(\mathrm{cmH}_{2} \mathrm{O}\right)\end{array}$ \\
\hline 1 day & 3.6 & 32 & 28 \\
1 month & 5.8 & 23 & 21
\end{tabular}

Coefficient of repeatability $=2$ SD of the differences between sessions.
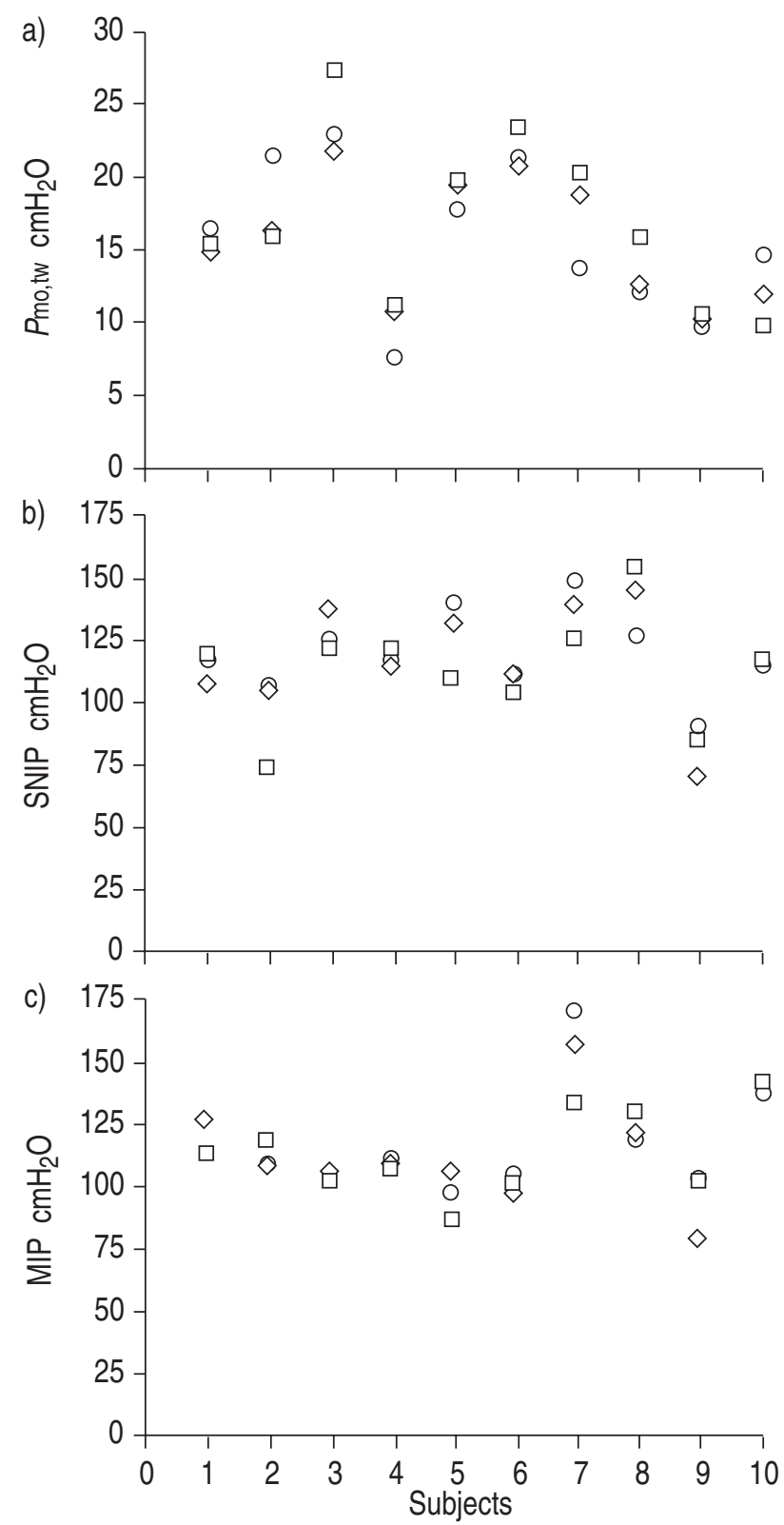

Fig. 1. - a) Median values of twitch mouth pressure $(P \operatorname{mo,tw})$ and maximal values of: b) sniff nasal inspiratory pressure (SNIP); and c) maximal inspiratory pressure (MIP). Values are from 10 subjects during session $1(\square)$, session 2 (after 1 day; $\gg$ ) and session 3 (after 1 month; $\bigcirc)$. 
The within session reproducibility is documented in table 2. The intraclass correlation coefficients were high and similar for the three methods, indicating that withinsubject variation was small in relation to between-subject variation. Over the three sessions, the mean within-session coefficient of variation was $12.6 \%$ for $P$ mo,tw, $6.0 \%$ for SNIP, and $5.7 \%$ for MIP.

The between-session reproducibility was expressed by the coefficient of repeatability, which indicates the value below which $95 \%$ of differences are expected (table 3 ). For $P$ mo,tw, the coefficient of repeatability was $3.6 \mathrm{cmH}_{2} \mathrm{O}$ at 1 day intervals, and $5.8 \mathrm{cmH}_{2} \mathrm{O}$ at 1 month intervals. For SNIP, the coefficient of repeatability was $32 \mathrm{cmH}_{2} \mathrm{O}$ at 1 day interval, and $23 \mathrm{cmH}_{2} \mathrm{O}$ at 1 month interval. For MIP, the coefficient of repeatability was $28 \mathrm{cmH}_{2} \mathrm{O}$ at 1 day interval and $21 \mathrm{cmH}_{2} \mathrm{O}$ at 1 month interval. The bet-weensession variability of each method in each subject is illustrated in figure 1 .

\section{Discussion}

As expected, the pressures generated by magnetic phrenic nerve stimulation were markedly lower than those produced by the volitional tests SNIP and MIP. Because of this difference of scale, the within-session reproducibility of the three tests was assessed by calculating the intraclass correlation coefficient. This index expresses the ratio of between-subject variation to total variation. The maximal value of the intraclass correlation coefficient is 1.0 and indicates perfect intrasubject reproducibility. It is considered that a test should have an intraclass correlation coefficient of at least 0.60 to be useful [13]. It can be seen from table 2 that the values of this index were high and similar for $P$ mo,tw, SNIP, and MIP, suggesting a similar usefulness of the three methods.

The within-session reproducibility of the three methods was also assessed by using coefficients of variation in order to compare the present results to previous studies. This is the first study of reproducibility of SNIP. As measured in the same subjects, the within-session reproducibility was equally good for SNIP and MIP, with a coefficient of variation of about $6 \%$, whereas it is generally reported at $8-9 \%$ for MIP $[15,16]$.

The within-session coefficient of variation of $P$ mo,tw was $12.6 \%$, which was higher than the $6.7 \%$ reported by WRAGG et al. [7] for twitch transdiaphragmatic pressure $(P \mathrm{di}, \mathrm{tw})$. Closure of the glottis may hamper the transmission of pressure swings from the alveoli to the mouth during phrenic nerve stimulation and may, therefore, adversely affect $P$ mo,tw reliability for assessing diaphragm strength $[9,10]$. In a preliminary study, we compared $P$ oes,tw, considered as reference, to three noninvasive measurements of inspiratory pressure during magnetic stimulation and found that $P$ mo,tw during a gentle exhalation from FRC showed excellent agreement with $P$ oes,tw. We think, therefore, like HamnEGaRD et al. [8], that transmission of pressure to the upper airways is not hindered by glottis closure with this technique. The increased variability that we ob-served could have resulted from technical errors during the magnetic stimulation. However, care was taken to en-sure constancy of stimulation by recording the diaphragmatic EMG response. Finally, variability could be related to changes in the lung volume at which the stimulation was applied, because lung volume affects $P$ di,tw $[17,18]$ and $P$ mo,tw [9]. We controlled the level of FRC by simple visual inspection to duplicate the conditions of the clinical field, and undetectable changes in FRC may have contributed to the variability that we observed. Furthermore, the level of lung volume attained by gentle exhalation from FRC was probably slightly different from one stimulation to another, although expiratory flow was very low and the duration of exhalation very short. Triggering the stimulator from the mouth pressure signal could reduce this part of variability, but it should be noted that in this setting the positive mouth pressure is determined by expiratory flow and the opening resistance rather than by lung volume.

For the three methods, the two way ANOVA showed that the variability was mostly between subjects, which reflects the well known interindividual variability of respiratory muscle strength [15]. Some variability was also due, to a lesser degree, to sessions and to an interaction between subjects and sessions. The latter finding reflects the fact that values increased across sessions for some subjects, and decreased for others, as illustrated by figure 1 . For the potential user of these methods, the key practical issue is how different will a measurement be if repeated at intervals. To provide this information, we expressed the between-session reproducibility by the coefficient of repeatability which indicates the value below which $95 \%$ of differences are expected [14]. Table 3 shows that for each time interval the coefficient of repeatability was similar for SNIP and MIP. Moreover, the coefficient of repeatability was slightly lower after 1 month than after 1 day, suggesting that reproducibility was not deteriorating with time. In contrast, for $P$ mo,tw, the coefficient of repeatability was slightly higher after 1 month than after 1 day. This loss of reproducibility was observed despite the same design using EMG control of stimulation in all sessions. The most likely explanation is a small variation in the spatial relationship between the stimulating coil and the phrenic nerves. It is conceivable that the positioning of the coil and of the neck was slightly less reproducible after a 1 month than after a 1 day interval.

In the present study, mean values of SNIP and MIP were similar, each of these techniques providing the highest pressure in half of the subjects. This is in agreement with a previous study by ULDRY and FITTING [4] on a larger number of healthy subjects, illustrating that these two manoeuvres complement one another. As expected, $P$ mo,tw was always markedly smaller than SNIP and MIP, and was similar to values reported by HAMNEGAARD et al. [8] using a similar technique [8]. Considering individual subjects, $P$ mo,tw represented a variable proportion of maximal volitional pressures, ranging $8-20 \%$ of SNIP and $9-23 \%$ of MIP. Thus, maximal volitional pressures cannot be re-liably extrapolated from $P$ mo,tw values, even in motivated healthy volunteers. This variability suggests differences in diaphragm recruitment during volitional manoeuvres [19]. Accordingly, combining volitional tests and nonvolitional phrenic nerve stimulation might be of interest to discriminate between diaphragm and other inspiratory muscles.

In summary, this study performed in healthy subjects showed a high and similar within session reproducibility for sniff nasal inspiratory pressure and maximal inspiratory pressure. The between-session reproducibility was also similar for sniff nasal inspiratory pressure and maximal inspiratory pressure, and did not decline after 1 month. This 
observation confirms the validity of sniff nasal inspiratory pressure as a noninvasive volitional test of inspiratory muscle strength [3-5]. In this study, anterior rhinoscopy was used in all subjects to exclude major anatomical abnormalities which could hinder the measurement of sniff nasal inspiratory pressure. In our experience, this is not necessary in clinical use of the test unless unexplained low values of sniff nasal inspiratory pressure are found. Measuring twitch mouth pressure during cervical magnetic stimulation represents a nonvolitional and noninvasive test of diaphragm strength. The within session reproducibility of twitch mouth pressure appeared sufficient to be clinically useful, but was lower than previously reported for the more invasive twitch transdiaphragmatic pressure. After 1 month, the reproducibility of twitch mouth pressure showed a slight decline which should be considered when this method is used to follow the course of an individual patient.

\section{References}

1. Black L, Hyatt R. Maximal respiratory pressures: normal values and relationship to age and sex. Am Rev Respir Dis 1969; 99: 696-702.

2. Polkey MI, Green M, Moxham J. Measurement of respiratory muscle strength. Thorax 1995; 50: 1131-1135.

3. Héritier F, Rahm F, Pasche P, Fitting JW. Sniff nasal inspiratory pressure. A noninvasive assessment of inspiratory muscle strength. Am J Respir Crit Care Med 1994; 150: $1678-1683$.

4. Uldry C, Fitting JW. Maximal values of sniff nasal inspiratory pressure in healthy subjects. Thorax 1995; 50: 371375.

5. Uldry C, Janssens JP, De Muralt B, Fitting JW. Sniff nasal inspiratory pressure in patients with chronic obstructive pulmonary disease. Eur Respir J 1997; 10: 1292-1296.

6. Similowski T, Fleury B, Launois S, Cathala HP, Bouche P, Derenne JP. Cervical magnetic stimulation: a new painless method for bilateral phrenic nerve stimulation in conscious humans. J Appl Physiol 1989; 67: 1311-1318.
7. Wragg S, Aquilina R, Moran J, et al. Comparison of cervical magnetic stimulation and bilateral percutaneous electrical stimulation of the phrenic nerves in normal subjects. Eur Respir J 1994; 7: 1788-1792.

8. Hamnegard CH, Wragg S, Kyroussis D, et al. Mouth pressure in response to magnetic stimulation of the phrenic nerves. Thorax 1995; 50: 620-624.

9. Yan S, Gauthier AP, Similowski T, Macklem PT, Bellemare F. Evaluation of human diaphragm contractility using mouth pressure twitches. Am Rev Respir Dis 1992; 145: 1064-1069.

10. Laghi F, Tobin MJ. Relationship between transdiaphragmatic and mouth twitch pressures at functional residual capacity. Eur Respir J 1997; 10: 530-536.

11. Wragg S, Hamnegard C, Road J, et al. Potentiation of diaphragmatic twitch after voluntary contraction in normal subjects. Thorax 1994; 49: 1234-1237.

12. Mador MJ, Magalang UJ, Kufel TJ. Twitch potentiation following voluntary diaphragmatic contraction. Am J Respir Crit Care Med 1994; 149: 739-743.

13. Chinn S. Repeatability and method comparison. Thorax 1991; 46: 454-456.

14. Bland JM, Altman DG. Statistical methods for assessing agreement between two methods of clinical measurement. Lancet 1986; i: 307-310.

15. Bellemare F. Strength of the respiratory muscles. In: Roussos C, ed. The Thorax. New York, Marcel Dekker, 1995; pp. 1161-1197. (Lenfant C, ed. Lung Biology in Health and Disease; vol 85).

16. Aldrich TK, Spiro P. Maximal inspiratory pressure: does reproducibility indicate full effort? Thorax 1995; 50: 40 43.

17. Smith J, Bellemare F. Effect of lung volume on in vivo contraction characteristics of human diaphragm. J Appl Physiol 1987; 62: 1893-1900.

18. Hamnegard CH, Wragg S, Mills G, et al. The effect of lung volume on transdiaphragmatic pressure. Eur Respir $J$ 1995; 8: 1532-1536.

19. Nava S, Ambrosino N, Crotti P, Fracchia C, Rampulla C. Recruitment of some respiratory muscles during three maximal inspiratory manoeuvres. Thorax 1993; 48: 702 707. 